\title{
Lack of Correlation between Severity of Clinical Symptoms, Skin Test Reactivity, and Radioallergosorbent Test Results in Venom-Allergic Patients
}

\author{
R.J. Warrington, $M B, B S, P h D, F R C P(C)$
}

\begin{abstract}
Purpose: To retrospectively examine the relation between skin test reactivity, venom-specific immunoglobulin $\mathrm{E}(\mathrm{IgE})$ antibody levels, and severity of clinical reaction in patients with insect venom allergy. Method: Thirty-six patients (including 15 females) who presented with a history of allergic reactions to insect stings were assessed. The mean age at the time of the reactions was $33.4 \pm 15.1$ years (range, 4-76 years), and patients were evaluated $43.6 \pm 90$ months (range, 1-300 months) after the reactions. Clinical reactions were scored according to severity, from 1 (cutaneous manifestations only) to 3 (anaphylaxis with shock). These scores were compared to scores for skin test reactivity ( 0 to 5 , indicating the log increase in sensitivity from $1 \mu \mathrm{g} / \mathrm{mL}$ to $0.0001 \mu \mathrm{g} / \mathrm{mL}$ ) and radioallergosorbent test (RAST) levels ( 0 to 4 , indicating venom-specific IgE levels, from undetectable to $>17.5$ kilounits of antigen per litre $[\mathrm{kUA} / \mathrm{L}])$.

Results: No correlation was found between skin test reactivity (Spearman's coefficient $=0.15, p=.377$ ) or RAST level (Spearman's coefficient $=0.32, p=.061$ ) and the severity of reaction. Skin test and RAST scores both differed significantly from clinical severity $(p<.05)$, but there was a significant correlation between skin test reactivity and RAST score $(p=.042)$. There was no correlation between skin test reactivity and time since reaction (Spearman's coefficient $=0.18, p=.294$ ) nor between RAST and time since reaction $(r=0.1353, p=.438)$. Elimination of patients tested more than 12 months after their reaction still produced no correlation between skin test reactivity $(p=.681)$ or RAST score $(p=.183)$ and the severity of the clinical reaction.

Conclusion: In venom-allergic patients (in contrast to reported findings in cases of inhalant IgE-mediated allergy), there appears to be no significant correlation between the degree of skin test reactivity or levels of venom-specific IgE (determined by RAST) and the severity of the clinical reaction.
\end{abstract}

R.J. Warrington-Departments of Medicine and Immunology, University of Manitoba, Winnipeg, Manitoba

Correspondence to: Dr. Richard Warrington, Section of Allergy and Clinical Immunology, GC319, 820 Sherbrook Street, Winnipeg, MB R3A 1R9; e-mail:

rwarrington@hsc.mb.ca

DOI 10.2310/7480.2006.00006
There is an understandable tendency on the part of patients (and probably also physicians) to assume that there is a clinical correlation between (1) the degree of skin test reactivity to an allergen on intradermal or epicutaneous prick testing for immediate hyposensitivity and (2) the level of clinical responsiveness to that allergen. A previous study examined this relationship for inhalant allergens and found a correlation between the severity of clinical symptoms and the degree of skin test reactivity (assessed by the size of the 
response) or the levels of specific immunoglobulin $\mathrm{E}$ (IgE) antibody (measured by radioallergosorbent test [RAST]). ${ }^{1}$ Other studies of venom and food allergies have not shown such a correlation..$^{2-5}$ In this analysis, investigators determined the relation between the above parameters for patients with a clinical history of immediate hypersensitivity to venoms of stinging insects by categorizing the clinical severity of the reaction to a sting, the levels of skin test reactivity to increasing concentrations of venoms, and specific IgE levels measured by RAST.

\section{Methods}

Thirty-six patients who were referred to the Allergy and Clinical Immunology Clinic at the Health Sciences Centre in Winnipeg for possible immunotherapy after having a systemic reaction to an insect sting were assessed. The group contained 15 females, and the mean age was $33.4 \pm$ 15.1 years ( $4-76$ years). The patients were evaluated $43.6 \pm 90$ months (median, 5.5 months; range, 1-300 months) after the reaction.

The patients were assessed in the following parameters:

1. Severity and characteristics of the clinical reaction, rated as follows ${ }^{6}$ :

a. Cutaneous manifestations only (hives, pruritus, or peripheral angioedema)

b. Upper- or lower-airway obstruction

c. Anaphylaxis with hypotension and shock necessitating resuscitation

2. Time (months) between the clinical reaction and the skin testing and RAST (done at the time of skin testing)

3. Skin test results after intradermal testing with venom in 10-fold dilutions from $1.0 \mu \mathrm{g} / \mathrm{mL}$ (positive-rated 1 on the skin testing scale) to $0.0001 \mu \mathrm{g} / \mathrm{mL}$ (positiverated 5 on the skin testing scale). Results of skin testing were compared to a positive (histamine $1.0 \mathrm{mg} / \mathrm{mL}$ ) and negative (saline) control, and a positive response had a wheal $\geq 5 \mathrm{~mm}$ and flare $\geq 10 \mathrm{~mm}$.
4. RAST (carried out in the Department of Clinical Chemistry, Health Sciences Centre, with Pharmacia assays)

No assays were carried out by Pharmacia CAP System RAST, and no patients suffered from significant gastrointestinal symptoms or cardiac/respiratory arrest.

Results were categorized as follows:

Negative: $0(<0.35$ kilounits of antigen per litre $[\mathrm{kUA} / \mathrm{L}])$

Low: $1(0.35-0.70 \mathrm{kUA} / \mathrm{L})$

Medium: 2 (0.7-3.5 kUA/L)

High: 3 (3.5-17.5 kUA/L)

Very high: 4 (> $17.5 \mathrm{kUA} / \mathrm{L})$

\section{Statistical Analysis}

Comparison of the three parameters (skin test, RAST, and clinical severity) was performed by Kruskal-Wallis one-way analysis of variance (ANOVA) on ranks, with SigmaStat 2.0 statistical software (Jandel Scientific). Correlation coefficients were determined by Spearman's rank order correlation, again with SigmaStat software. Power was assessed by $P S$ power and sample size calculation software (Dupont and Plummer, freeware, 1997). Graphic analysis was done with DPlot 2.0.0.3 software (HydeSoft Computing).

\section{Results}

Of the 36 patients assessed, 16 had a reaction with only cutaneous manifestations, 13 had upperor lower-airway obstruction, and 6 had anaphylaxis with cardiovascular collapse. None had significant gastrointestinal symptoms.

Twenty-seven patients were assessed within 12 months of their reaction, and the remaining nine patients were seen 14 to 300 months after the reaction to venom (Table 1 ).

As determined by the scoring system described previously, the mean score for skin tests was $2.806 \pm 1.064$ standard deviation $(\mathrm{SD})$, the mean 
Table 1 Demographics of Patient Population

\begin{tabular}{|c|c|c|c|c|c|c|}
\hline Number & Sex & Age (yr) & Skin Test & $R A S T$ & $\begin{array}{c}\text { Clinical } \\
\text { Reaction }\end{array}$ & $\begin{array}{c}\text { Months since } \\
\text { Reaction }\end{array}$ \\
\hline 1 & $\mathrm{~F}$ & 47 & 3 & 4 & 1 & 1 \\
\hline 2 & $\mathrm{M}$ & 33 & 3 & 1 & 1 & 2 \\
\hline 3 & $\mathrm{~F}$ & 14 & 4 & 3 & 2 & 204 \\
\hline 4 & M & 21 & 1 & 2 & 2 & 10 \\
\hline 5 & $\mathrm{~F}$ & 41 & 0 & 3 & 1 & 2 \\
\hline 6 & $\mathrm{~F}$ & 49 & 3 & 2 & 1 & 14 \\
\hline 7 & M & 25 & 1 & 3 & 1 & 84 \\
\hline 8 & $\mathrm{M}$ & 21 & 4 & 4 & 3 & 1 \\
\hline 9 & $\mathrm{M}$ & 4 & 4 & 4 & 3 & 240 \\
\hline 10 & $\mathrm{~F}$ & 20 & 3 & 3 & 1 & 4 \\
\hline 11 & M & 18 & 2 & 3 & 1 & 5 \\
\hline 12 & $\mathrm{~F}$ & 23 & 5 & N/A & 1 & 276 \\
\hline 13 & $\mathrm{M}$ & 26 & 3 & 1 & 2 & 2 \\
\hline 14 & $\mathrm{~F}$ & 33 & 4 & 4 & 2 & 1 \\
\hline 15 & $\mathrm{~F}$ & 46 & 2 & 2 & 1 & 12 \\
\hline 16 & $\mathrm{M}$ & 49 & 4 & 3 & 2 & 30 \\
\hline 17 & $\mathrm{M}$ & 59 & 2 & 3 & 2 & 2 \\
\hline 18 & $\mathrm{~F}$ & 36 & 2 & 3 & 2 & 12 \\
\hline 19 & M & 37 & 3 & 3 & 2 & 18 \\
\hline 20 & $\mathrm{M}$ & 28 & 4 & 4 & 1 & 300 \\
\hline 21 & $\mathrm{~F}$ & 40 & 3 & 3 & 1 & 3 \\
\hline 22 & $\mathrm{M}$ & 6 & 3 & 1 & 1 & 3 \\
\hline 23 & M & 33 & 2 & 2 & 1 & 276 \\
\hline 24 & $\mathrm{~F}$ & 46 & 2 & 3 & 1 & 3 \\
\hline 25 & M & 35 & 2 & 2 & 3 & 6 \\
\hline 26 & $\mathrm{M}$ & 41 & 3 & 3 & 3 & 6 \\
\hline 27 & $\mathrm{~F}$ & 76 & 1 & 4 & 3 & 1 \\
\hline 28 & $\mathrm{M}$ & 24 & 4 & 4 & 2 & 1 \\
\hline 29 & $\mathrm{M}$ & 16 & 2 & 2 & 2 & 3 \\
\hline 30 & $\mathrm{~F}$ & 35 & 3 & 2 & 1 & 1 \\
\hline 31 & $\mathrm{~F}$ & 42 & 3 & 4 & 2 & 2 \\
\hline 32 & $\mathrm{M}$ & 29 & 3 & 4 & 2 & 12 \\
\hline 33 & $\mathrm{M}$ & 28 & 3 & 1 & 6 & 6 \\
\hline 34 & $\mathrm{~F}$ & 20 & 3 & 3 & 2 & 2 \\
\hline 35 & $\mathrm{M}$ & 46 & 4 & 3 & 1 & 12 \\
\hline 36 & $\mathrm{M}$ & 56 & 3 & 4 & 3 & 12 \\
\hline
\end{tabular}

RAST $=$ radioallergosorbent test.

score for RAST was $2.857 \pm 0.974$, and the mean score for clinical severity was $1.833 \pm 1.028$.

By ANOVA, the skin test score was significantly different from the clinical severity score $(p<.05$, power $=0.988)$. The RAST score was also significantly different from the clinical severity score $(p<.05$, power $=0.996)$. However, the RAST score did not differ significantly from the skin test score $(p>.05)$.
As determined by Spearman's rank order correlation for data not normally distributed, there was a significant correlation between skin test score and RAST score $(r=0.346, p=.042$, power $=0.626)$ but no correlation between clinical severity and either skin test score $(r=0.0957, p=.576)$ or RAST score $(r=0.246, p=.152)$. These results are shown graphically in a three-dimensional representation in Figure 1, where it can be seen that the 


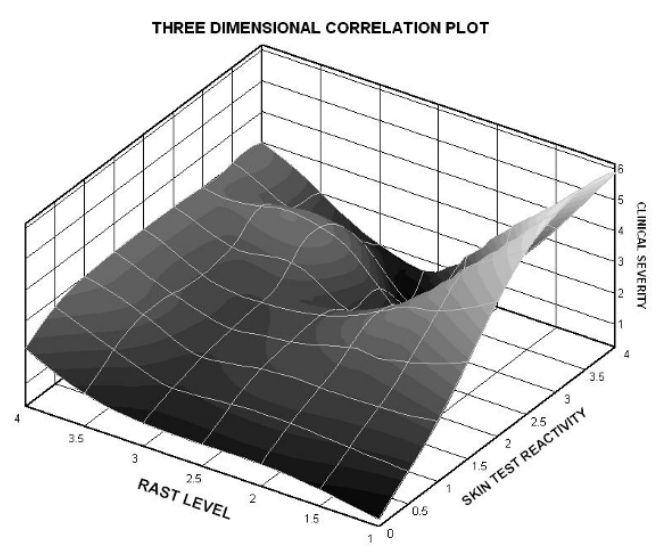

Figure 1 Three-dimensional plot of skin test, RAST, and clinical severity scores of 36 patients with stinging-venom allergy (as a surface plot).

clinical severity score tends to increase at lower RAST scores and in the middle range of skin test scores.

There was no correlation between skin test reactivity and time since reaction $(r=0.18$, $p=.294)$ or RAST score and time since reaction $(r=0.183, p=.438)$.

Even if patients who were tested more than 12 months after their reaction were excluded, there was still no correlation between skin test reactivity $(p=.681)$ or RAST score $(p=.183)$ and the severity of the clinical reaction.

\section{Discussion}

At this time, there are three methods by which allergy to venom from stinging insects can be assessed: (1) determination of the clinical severity of the reaction, (2) skin tests to measure reactivity, and (3) RAST or CAP System assay to measure specific IgE. From a linear thinking perspective, it might be assumed (excluding loss of reactivity with time as a factor) that a correlation exists between these three parameters; this does not, however, appear to be the case.

Patrizzi and colleagues evaluated skin tests and estimations of specific IgE antibodies by RAST in patients with bee-sting allergy. ${ }^{7}$ The results corresponded in $82 \%$ of the patients. Hoffman com- pared RAST and skin test results for five venoms in 60 patients with histories of stinging-insect allergy; 48 patients with positive skin test results showed an $88 \%$ correlation of RAST and skin test scores. ${ }^{8}$ Egner and colleagues reported that the level of IgE antibody to venom does not reliably reflect the severity of the last reaction to a sting in patients with double positive reactivity to stings both from honeybees and from Vespula species. ${ }^{9}$

Mosbech, in a comparative study of venomallergic patients, found a positive correlation $(p<.05)$ between the results of skin-prick tests and specific IgE against venoms. For patients allergic to yellowjacket stings, there was also a correlation between the severity of symptoms after the sting and the size of the skin-prick test reaction to venom. ${ }^{2}$ In contrast, Nittner-Marszalska and colleagues found that for patients with allergy to the venom of insects of the order Hymenoptera, there was no correlation between the size of the skin test reaction, the class of venom-specific IgE level (by fluorescent allergosorbent technique [FAST]), and the results of basophil histamine release. In addition, no relation was found between the results of these tests and the severity of the sting reactions as measured on the Mueller scale. ${ }^{3}$

In this analysis of 36 patients with immediate hypersensitivity reactions to stinging-insect venoms, no correlation was found between the degree of skin test reactivity or RAST score and the severity of the clinical reaction. The absence of a significant correlation persisted when individuals who were assessed more than 12 months after their clinical reaction were excluded from the analysis. A correlation was found between the degree of skin test reactivity and venom-specific IgE levels measured by RAST.

The fact that skin test reactivity and specific IgE levels measured by RAST are not predictors of the clinical severity of a reaction is apparently not unique to venom allergy. Rosario and Vilela found that when using quantitative skin-prick tests with an average endpoint allergen concentration of 50 allergen units (AU) on atopic asthmatic patients, the mean wheal diameter was not significantly greater for severely asthmatic patients than for mildly asthmatic patients. ${ }^{4}$ In patients with nut allergy, Clark and Ewan ${ }^{5}$ found 
no correlation between skin-prick test wheal size and the graded severity of the worst reaction for all nuts combined or for peanut, hazelnut, almond, and walnut. For the CAP System specific IgE levels, there was no correlation for all nuts, so the size of SPT or CAP System levels did not predict between minor urticaria and anaphylaxis.

These results indicate that specific IgE level as assessed by either skin testing or serum $\operatorname{IgE}$ is only one determining parameter in the induction of anaphylaxis and that other factors must play a role. Such factors could be mast-cell mass, mastcell stability, concomitant medications, number of stings or amount of venom injected, and levels of blocking antibodies. Although it is tempting to assume so, the size of the skin test reaction to an allergen or the level of specific IgE does not necessarily predict clinical reactivity.

In contrast to the above studies, Souille and colleagues ${ }^{1}$ assessed 59 asthmatic children by skin-prick tests, RAST (specific $\operatorname{IgE}$ ), and bronchial provocation with common inhalants. They found a significant connection between the results of the three tests. However, the concordance level was only moderate, i.e., not greater than $68 \%$. In comparison with bronchial provocation, prick testing and RAST respectively yielded numerous false-positive and false-negative results. This is perhaps not surprising, given the problem of allergen sensitization without clinical reactivity and the reduced sensitivity of RAST when compared with skin testing. In this study, the negative predictive value of skin-prick tests was considered satisfactory, and the most discriminatory threshold for the positive RAST result was the class-3 response. This would suggest that correlation between the magnitude of skin-prick tests or RAST and bronchial challenge was not good.

van der Linden and colleagues reported on insect-sting challenge of 324 subjects with a previous anaphylactic reaction to yellowjacket and honeybee stings and found that a recurrence of anaphylaxis was observed in $25 \%$ of those sensitive to yellowjacket stings and $52 \%$ of those sensitive to honeybee stings. ${ }^{10}$ Even more striking were the findings of van Halteren and colleagues, who described 348 patients with a previous history of anaphylaxis to stings and with mild or no symptoms on in-hospital sting challenge; 129 of these subjects subsequently were accidentally stung in the field, and 110 had only local reactions whereas 6 patients experienced serious manifestations. ${ }^{11}$ Therefore, previous history of a severe response to a sting is not predictive of a subsequent serious reaction to accidental sting challenge. Meanwhile, two-thirds of individuals who die from a sting reaction have no previous history of their allergy. ${ }^{12}$

\section{Conclusion}

Allergic reactivity is a complex phenomenon that is not explainable simply on the basis of the presence of immunoglobulin $\mathrm{E}$ antibodies. The difficulty is to devise methods of determining who among individuals with equivalent levels of sensitization is at risk of a serious reaction.

\section{References}

1. Souille B, et al. Bronchial provocation tests in 59 asthmatic children. Comparison with skin tests and serum allergens. Rev Mal Respir 1987;4: 225-30.

2. Mosbech H. Insect allergy. A comparative study including case histories and immunological parameters. Allergy 1984;39:543-9.

3. Nittner-Marszala M, et al. Evaluation of diagnostic value of skin test, venom specific IgE antibodies and basophil histamine release test in Hymenoptera allergy. Pneumonal Alergol Pol 1993;61:346-51.

4. Rosario NA, Vilela MM. Quantitative skin prick tests and serum IgE antibodies in atopic asthmatics. J Investig Allergol Clin Immunol 1997;7:40-5.

5. Clark AT, Ewan PW. Interpretation of tests for nut allergy in one thousand patients, in relation to allergy or tolerance. Clin Exp Allergy 2003;33:1041-5.

6. Ring J, et al. History and classification of anaphylaxis. Novartis Found Symp 2004;257: 6-16.

7. Patrizzi R, et al. Comparison of skin tests and RAST for the diagnosis of bee-sting allergy. Allergy 1979;34:249-56. 
8. Hoffman DR. Comparison of the radioallergosorbent test to intradermal skin testing in the diagnosis of stinging insect venom allergy. Ann Allergy 1979;43:211-3.

9. Egner W, et al. The frequency and clinical significance of specific IgE to both wasp (Vespula) and honey-bee (Apis) venoms in the same patient. Clin Exp Allergy 1998;28:26-34.

10. van der Linden PW, et al. Insect-sting challenge in 324 subjects with a previous anaphylactic reaction: current criteria for insect venom hypersensitivity do not predict the occurrence and severity of anaphylaxis. J Allergy Clin Immunol 1994;94:151-9.

11. van Halteren HK, et al. Hymenoptera sting challenge of 348 patients: relation to subsequent field stings. J Allergy Clin Immunol 1996;97:1058-63.

12. Pumphrey R. Anaphylaxis: can we tell who is at risk of a fatal reaction? Curr Opin Allergy Clin Immunol 2004;4:285-90. 